\title{
Lesión por inhalación, qué hay de nuevo
}

Inhalation injury, what is again

Lesão por inalação, o que há de novo

Marco Antonio Garnica Escamilla, ${ }^{*}$ Karen Itzel González Martínez, ${ }^{\ddagger}$ Oscar Miguel Marín Landa, ${ }^{*}$ Elsa Carolina Laredo Sánchez, ${ }^{\star}$ Martín de Jesús Sánchez Zúñiga, ${ }^{*}$ Raúl Carrillo Esper*

\section{RESUMEN}

La lesión por inhalación o quemadura de la vía aérea se produce en la mucosa de la vía aérea o en el parénquima pulmonar, debido a acción directa de la transferencia de calor, así como por la inhalación de humo o productos químicos de la combustión incompleta de los compuestos orgánicos e inorgánicos. La incidencia de quemadura de la vía aérea es de 5 a $35 \%$ e incrementa $2 \%$ si la superficie corporal quemada es menor a $20 \%$ hasta $55 \%$ si la superficie corporal quemada es mayor a $80 \%$, de igual forma incrementa la mortalidad de 20 a $30 \%$. Aun cuando se realice un manejo perioperatorio efectivo que incluya un adecuado manejo de líquidos con una reanimación temprana, una pronta escisión quirúrgica del tejido quemado, adecuado uso de técnicas ventilatorias la inhalación de humo se merece un manejo dirigido y específico ya que por su asociación con quemadura, mueren por esta causa. El diagnóstico de lesión por inhalación es difícil y no existe proporción entre la gravedad de la quemadura cutánea y la lesión pulmonar.

Palabras clave: Lesión por inhalación, quemadura de vía aérea, productos químicos de la combustión, heparina, vía aérea difícil, broncoscopia flexible.

\section{ABSTRACT}

Inhalation injury or airway burn is the lesion that occurs in the airway mucosa or in the lung parenchyma, due to the direct action of heat transfer, as well as the inhalation of smoke or chemicals from the airway. Incomplete combustion of organic and inorganic compounds. The incidence of Airway Burn is 5 to 35\% and increases $2 \%$ if the Burned body surface is less than $20 \%$ up to $55 \%$ if the burned body surface is greater than $80 \%$, likewise increases the mortality of 20 to $30 \%$. Even when effective perioperative management is carried out, including adequate fluid management with early resuscitation, prompt surgical excision of burned tissue, adequate use of ventilatory techniques, smoke inhalation deserves targeted and specific management because of its association with burn, die for this cause. The diagnosis of inhalation injury is difficult and there is no proportion between the severity of the skin burn and the lung injury.

Keywords: Inhalation injury, airway burn, chemical products of combustion, heparin, difficult airway, flexible bronchoscopy.

\section{RESUMO}

A lesão por inalação ou queimaduras das vias aéreas é a lesão que ocorre na mucosa das vias aéreas ou no parênquima pulmonar, devido à ação direta da transferência de calor, bem como à inalação de fumaça ou produtos químicos de combustão incompleta de compostos orgânicos e inorgânicos. A incidência de queimadura das vias aéreas é de 5 a $35 \%$ e aumenta em $2 \%$ se a superfície corporal queimada for inferior a 20 até $55 \%$ se a superfície corporal queimada for maior que $80 \%$, da mesma forma aumenta a mortalidade de 20 a $30 \%$. Mesmo quando é realizado manejo perioperatório eficaz, que inclui manejo adequado de fluidos com reanimação precoce, excisão cirúrgica imediata do tecido queimado, uso adequado de técnicas ventilatórias, a inalação de fumaça merece manejo direcionado e específico, pois, devido à sua associação com uma queimadura, eles morrem por essa causa. O diagnóstico de lesão por inalação é difícil e não há proporção entre a gravidade da queimadura na pele e a lesão pulmonar.

Palavras-chave: Lesão por inalação, queimadura das vias aéreas, produtos químicos de combustão, heparina, via aérea difícil, broncoscopia flexível.

\footnotetext{
* Centro Nacional de Investigación y Atención de Quemados. Instituto Nacional de Rehabilitación, «Luis Guillermo Ibarra Ibarra».

‡ Hospital General de Mexicali

Recepción: 14/08/2019. Aceptación: 18/12/2019.

Citar como: Garnica EMA, González MKI, Marín LOM, Laredo SEC, Sánchez ZMJ, Carrillo ER. Lesión por inhalación, qué hay de nuevo. Med Crit. 2021;35(4):206-215. https://dx.doi.org/10.35366/101160

www.medigraphic.com/medicinacritica
}

\section{INTRODUCCIÓN}

El término de lesión por inhalación de humo es un término no específico, abarca distintos tipos de lesión: térmica, química e intoxicación sistémica; esta lesión puede clasificarse a su vez en lesión de la vía aérea superior, lesión de la va aérea inferior y lesión del parénquima pulmonar (toxicidad sistémica). ${ }^{1}$

La extensión del daño por la inhalación depende del escenario en que se produjo el fuego (espacio abierto y/o cerrado), de la fuente de origen, la temperatura, la concentración y la solubilidad de los humos y gases producidos por la combustión, la duración del fuego y el tiempo de exposición de las víctimas. ${ }^{1}$

El manejo de la lesión por inhalación es un desafío que interrelaciona la aplicación del conocimiento de la fisiopatología al manejo perioperatorio, con desarrollo de destrezas y habilidades para la modificación del impacto de la respuesta orgánica térmica, no en forma aislada sino como una concentración de efectos determinados por la respuesta inflamatoria sistémica, con cambios y alteraciones importantes en las condiciones de la vía aérea, función respiratoria, cardiaca, hepática, de nutrición y metabólica; anormalidades hidroeletrolíticas, de termorregulación y renales, así como de los resultados obtenidos en la atención, reanimación y manejo inicial pre hospitalario que será influencia determinante en el riesgo de inmunosupresión. ${ }^{2}$

El objetivo del presente trabajo, tomando en cuenta la incidencia y la mortalidad relacionada con la lesión por inhalación, es poder dar una pauta inicial para la sospecha y reconocimiento temprano de esta patología, logrando así instaurar un manejo integral, ya que el personal médico de primer contacto, en este caso urgenciólogos, anestesiólogos y médicos intensivistas, tiene como reto la toma de decisiones oportunas y adecuadas en la evaluación, manejo y control de la vía aérea.

\section{PRINCIPIOS GENERALES}

Las quemaduras de siniestros no accidentales en $90 \%$ de los casos se producen por la exposición repetitiva a actividades de riesgo debido a la falta de cultura preventiva de la población, la pobreza, la falta de normatividad legal y la falta de control de violencia social. La literatura médica y los datos epidemiológicos registrados en 
los sistemas estadísticos electrónicos de la Secretaría de Salud de México demuestran que los «accidentes" y las quemaduras son enfermedades prevenibles que generan alta mortalidad y discapacidad, así como altos costos de atención médica integral. ${ }^{3}$

En el Hospital de Shrinners de Galveston, Texas, durante el periodo comprendido entre 1989 y 2008, fueron hospitalizados 5,260 niños quemados con sólo 145 defunciones $(2.8 \%)$. Las causas de quemaduras fueron: $67 \%$ por fuego, $23 \%$ por escaldadura y $11 \%$ por otras razones. La edad promedio de dichos pacientes fue de 7.3 años de edad. El promedio de superficie corporal quemada de los pacientes fue de $55 \%$. En $34 \%$ de los pacientes se presentó lesión por inhalación. Los principales determinantes de supervivencia fueron superficie corporal quemada de $60 \%$ y lesión por inhalación. ${ }^{3}$

La incidencia de insuficiencia respiratoria es significativa después de lesiones por inhalación, con hipoxemia, neumonía e insuficiencia respiratoria con una duración prolongada de soporte ventilatorio y hospitalizaciones extendidas. ${ }^{4}$

El diagnóstico precoz se puede lograr mediante el reconocimiento de los factores de riesgo revelados por la historia y examen físico y confirmados por procedimientos diagnósticos. ${ }^{5}$

\section{FACTORES DE RIESGO}

Los factores de riesgo, como ya los mencionamos anteriormente, serán revelados por la historia clínica completa, dentro de éstos nos vemos obligados a investigar el lugar donde ocurrió el incidente (espacio cerrado y/o abierto), el tiempo de exposición, pérdida del estado de alerta, fuente de origen, temperatura, concentración y solubilidad de los gases y humos producidos por la combustión así como la edad del paciente. ${ }^{1}$

\section{FISIOPATOLOGÍA DE LA LESIÓN POR INHALACIÓN}

\section{Primera fase:}

En la primera fase de la lesión por inhalación, el paciente puede presentar intoxicación por monóxido de carbono que se produce en la combustión de material orgánico o sintético. El monóxido de carbono se une a la hemoglobina y al sistema mitocondrial de citocromo oxidasa, provocando deterioro del metabolismo aeróbico. Los síntomas se relacionan directamente a las concentraciones de carboxihemoglobina. El cianuro es otro agente asfixiante que se libera por la combustión de algunos materiales plásticos como poliuretanos, poliacrilonitrilo y goma de acrocianato. La intoxicación provoca asfixia por inhibición de la actividad intracelular de la citocromo oxidasa. ${ }^{2}$

\section{Segunda fase:}

Ocurre entre las 24 a 96 horas después de la lesión y resulta del daño del parénquima pulmonar causado por la irritación química. Se caracteriza por edema de la vía aérea, edema pulmonar, traqueobronquitis, atelectasia, incremento de resistencia de la vía aérea y disminución de compliance y volúmenes pulmonares. ${ }^{2}$

Con base en la localización primaria de la lesión, ésta se clasifica en lesión de la vía aérea superior, del sistema traqueo bronquial o el parénquima pulmonar:

- Lesión de la vía aérea superior (por encima de las cuerdas vocales): es una lesión térmica debida al intercambio de calor eficiente en la orofaringe y nasofaringe. La lesión inmediata produce eritema, ulceraciones y edema, se ve afectada la función ciliar que afecta los procesos fisiológicos de eliminación de las vías respiratorias, lo que aumenta el riesgo de infecciones bacterianas durante varias semanas, además la producción incrementada de secreciones espesas puede causar oclusión de la vía aérea distal, atelectasia y afección en el intercambio de gases. ${ }^{6,7}$

- Lesión del árbol traqueobronquial: generalmente es causada por químicos en el humo, así como la inhalación tóxica de gases nocivos, líquidos y el fuego directo de las vías respiratorias. El área traqueobronquial está inervada por las terminaciones nerviosas vasomotoras y sensoriales, así, la inhalación de humo estimula estos nervios para liberar neuropéptidos que se encargarán de inducir broncoconstricción y óxido nítrico sintetasa (NOS) con la cual se generarán especies reactivas de oxígeno (ROS). Estos neuropéptidos pueden funcionar como taquiquininas induciendo una respuesta inflamatoria con los efectos posteriores de la broncoconstricción, el aumento de la permeabilidad vascular y la vasodilatación. Estos factores potencian el daño celular local y la pérdida de vasoconstricción pulmonar hipóxica, que hace que el flujo sanguíneo bronquial aumente muchas veces, el aumento del flujo bronquial libera leucocitos polimorfonucleares activados y citoquinas en el pulmón, lo que aumenta la respuesta inflamatoria. ${ }^{6,7}$

- Lesión del parénquima pulmonar: la diferencia de tiempo desde la lesión inicial a la aparición de una disminución de la presión arterial de oxígeno hasta la proporción de la fracción inspirada de oxígeno se correlaciona con la gravedad de la lesión pulmonar, un tiempo más rápido se asocia con lesiones más graves. La lesión del parénquima pulmonar se caracteriza por atelectasia y colapso alveolar que da como resultado un aumento del flujo de fluido transvascular, una disminución del agente tensoactivo y una pérdida de vasoconstricción y por lo tanto alteración en la oxigenación. Además de un desequilibrio grave en la 
Tabla 1: Signos y síntomas en la lesión inhalatoria.

- Quemaduras faciales (área oro-nasal)

- Quemadura de vibrisas nasales

- Tos con esputo carbonáceo

- Disfonía, disnea

- Dolor torácico, hemoptisis

- Cianosis, taquipnea

- Estridor, broncoespasmo

- Asimetría en movimientos respiratorios

Tabla 2: Indhicadores de lesión de la vía aérea.

- Dificultad respiratoria o taquipnea

- Quemaduras que involucran cara y cuello

- Circunferenciales de tórax y abdomen

- Cambio de voz, ronquera

- Tos

- Disfagia

- Sialorrea

- Esputo carbonáceo

hemostasia alveolar y la disminución de la actividad fibrinolítica, con deposición masiva de fibrina, lo que causa un desequilibrio entre la ventilación-perfusión. , $^{6,7}$

La obstrucción de las vías respiratorias y atelectasia aumenta el riesgo de neumonía. El riesgo de neumonía aumenta debido a la función deteriorada de los macrófagos alveolares, los leucocitos polimorfonucleares y los mecanismos de eliminación mucociliar.

La toxicidad sistémica es secundaria al efecto directo de la lesión por inhalación que se produce al respirar sustancias tóxicas formadas por combustión o pirólisis. Los dos gases más relevantes asociados con una mayor morbilidad y mortalidad son el monóxido de carbono y el cianuro. ${ }^{6}$

Dentro de la fisiopatología de la lesión por inhalación podemos encontrar otra clasificación en la literatura, la lesión anóxica que está dada por disminución de oxígeno y aumento de dióxido de carbono, exposiciones durante un minuto potencian el efecto de otros productos químicos y la Lesión Química que es secundaria a sustancias tóxicas que se producen de la combustión o descomposición química de la particular. ${ }^{8}$

\section{MANIFESTACIONES CLÍNICAS}

Las manifestaciones clínicas de la lesión por inhalación son variadas y van a depender de la localización y de las sustancias químicas producidas de la combustión (Tablas 1 y 2). ${ }^{1,2}$

Los síntomas generales que se pueden presentar son mareo, náusea o vómito. La intoxicación por monóxido de carbono se debe sospechar en cualquier paciente que presente inhalación de humo hasta que se excluya por un nivel de carboxihemoglobina normal medida por CO-oximetría (Tabla 3).1,2,9
El inicio de los síntomas puede ser progresivo o brus$\mathrm{co}$, aumentando el riesgo vital. En los casos progresivos, será precedido por ronquera y disnea, mientras que en los bruscos se presentará disnea y luego paro respiratorio posterior a la obstrucción de la vía aérea superior. ${ }^{10}$

Inicialmente, puede existir ausencia de síntomas respiratorios, retrasándose la aparición de éstos, hasta 18 a 72 horas después de la quemadura, presentarse tos irritativa o desarrollarse una insuficiencia respiratoria 24 a 48 horas más tarde por aparición de atelectasias, disminución de la compliance pulmonar o dificultad para eliminar las abundantes secreciones (Tabla 4). ${ }^{11}$

El edema de la vía aérea superior suele resolverse en cuatro a cinco días, la exploración laringotraqueal es útil en estos casos para observar lesiones de la mucosa de la vía aérea superior o cuerdas vocales, pero lamentablemente no nos informa del compromiso traqueobronquial. ${ }^{10}$

La irritación de la capa mucosa de las vías aéreas puede persistir varios días, produciendo tos y broncorrea. En tres o cuatro días la mucosa afectada se convierte en necrótica, desprendiéndose y aumentando la viscosidad de las secreciones. ${ }^{10}$

\section{DIAGNÓSTICO DE LESIÓN POR INHALACIÓN; UN RETO PARA EL MÉDICO}

El diagnóstico de la lesión por inhalación puede sospecharse sobre la base de los hallazgos clínicos en el contexto de exposición al humo, pero el diagnóstico

Tabla 3: Correlación entre síntomas clínicos y porcentaje de monóxido de carbono en sangre.

\begin{tabular}{|c|c|}
\hline $\mathrm{CO}_{2}(\%)$ & Síntomas \\
\hline $0-10$ & $\begin{array}{l}\text { Ninguno (en pacientes con cardiopatía coronaria puede } \\
\text { presentarse angina) }\end{array}$ \\
\hline $10-20$ & $\begin{array}{l}\text { Ligera cefalea, angina de esfuerzo, disnea con ejercicio } \\
\text { enérgico }\end{array}$ \\
\hline 20-30 & Cefalea palpitante, disnea con ejercicio moderado \\
\hline $30-40$ & $\begin{array}{l}\text { Cefalea intensa, náusea y vómito, debilidad, alteraciones } \\
\text { visuales, alteración del raciocinio }\end{array}$ \\
\hline $40-50$ & Síncope, taquicardia, taquipnea y disnea de reposo \\
\hline $50-60$ & Coma, convulsiones, respiración de Cheyne-Stokes \\
\hline $60-70$ & Compromiso de la función cardiorrespiratoria \\
\hline $70-80$ & Muerte \\
\hline
\end{tabular}

Grande CM, Myers RAM. Hiperbaric medicine: a trauma perspective. In: Stene JK, Grande CM, editors: Trauma anesthesia. Baltimore; Williams $\delta$ Wilkins. 1991, pp. 368-406.

Tabla 4: Hallazgos radiográficos en lesión por inhalación.

\begin{tabular}{ll}
\hline 0 & Normal \\
1 & Edema perivascular o peribronquial \\
2 & Edema alveolar o intersticial en el tercio inferior de los campos pulmonares \\
3 & Si se extiende a los dos tercios inferiores de los campos pulmonares \\
4 & Si ocupa la totalidad del campo pulmonar \\
\hline
\end{tabular}




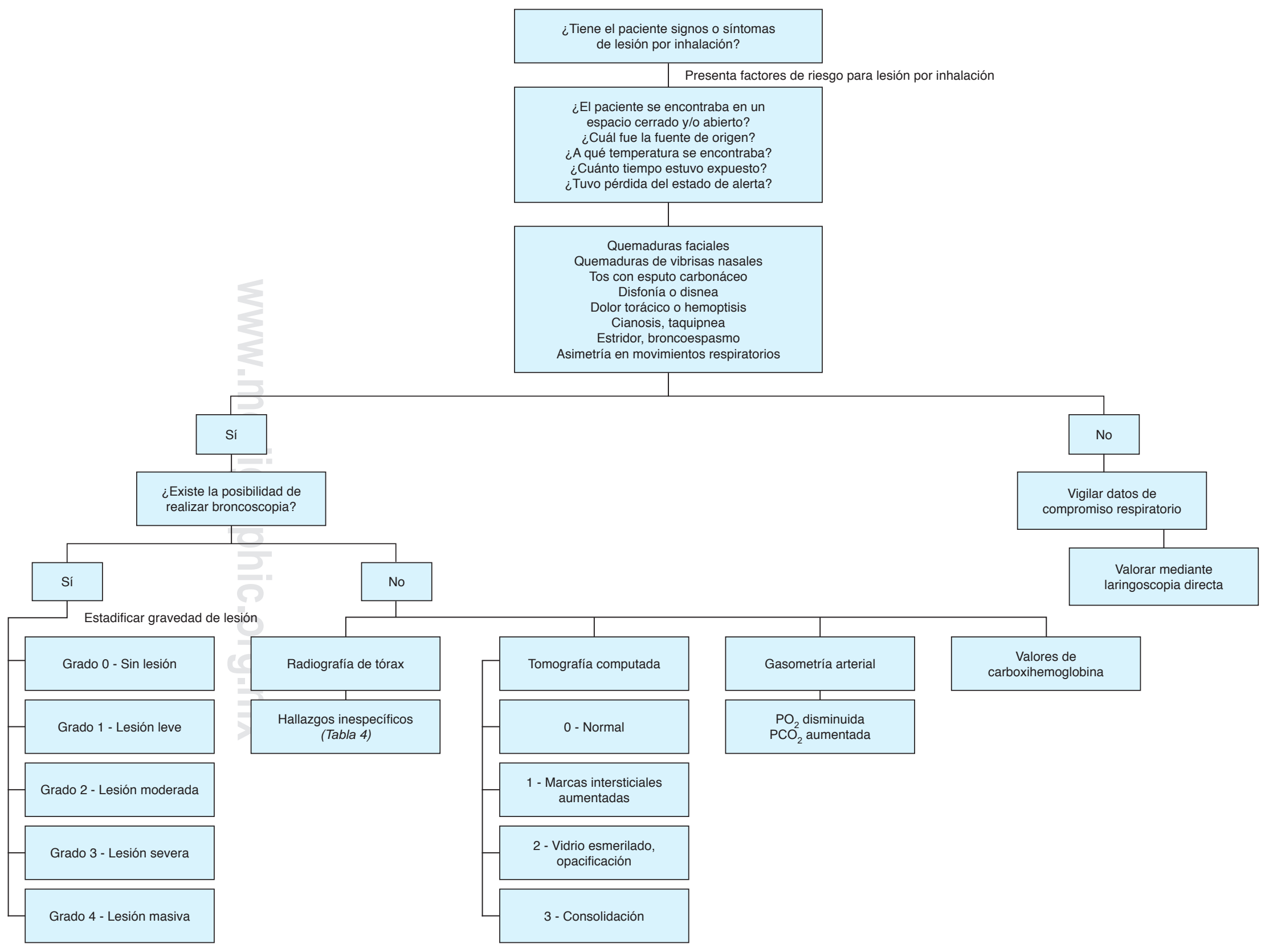

Algoritmo 1: Diagnóstico del paciente con lesión por inhalación. 
definitivo se basa en el examen directo de las vías respiratorias. Existen varios estudios que nos orientan al diagnóstico desde la clínica y el simple hecho de sospechar la lesión hasta el diagnóstico más específico y el estándar de oro con broncoscopia flexible, esta entidad clínica resulta ser un reto para el médico debido a que no en todos los centros de atención contamos con el material necesario para llegar a un diagnóstico y en muchas ocasiones nos basamos precisamente en manifestaciones clínicas, lo que es difícil ya que encontraremos pacientes en los que a pesar de tener compromiso de la vía aérea se encontrarán completamente asintomáticos. En este apartado citaremos los estudios complementarios que podemos utilizar (Algoritmo 1).

\section{Radiografía, lo más disponible pero poco eficaz}

Es un estudio está disponible en la mayoría de los centros de atención; sin embargo, no posee mucha utilidad en la fase aguda. No tiene poder diagnóstico y posee un alto porcentaje de falsos negativos (92\%). El tiempo de aparición de los hallazgos son de 24 a 36 horas. $^{8}$

Peitzman y colaboradores emplean una escala semicuantitativa para valorar el grado de afectación pulmonar (Tabla 4). Sin embargo, pasadas las 48 horas posteriores a la quemadura, puede ser difícil dilucidar si los hallazgos patológicos encontrados en la radiografía de tórax se deben a la lesión por inhalación, a la infección pulmonar, al edema pulmonar hidrostático o al SDRA. ${ }^{12}$

\section{Tomografía computarizada de tórax}

Se considera otro de los métodos útiles para evaluar la gravedad de la lesión. Se desarrolló un sistema de puntuación para la gravedad de los hallazgos en la tomografía. $^{13}$

Esta clasificación se basó en un estudio retrospectivo de todos los pacientes admitidos en el Instituto del Ejército de los Estados Unidos de Surgical Research Burns Center entre 2002 y 2008 con tomografía de tórax dentro de las primeras 24 horas de admisión. La hipótesis en este estudio fue que una tomografía computarizada junto con la broncoscopia se puede utilizar como una herramienta de pronóstico para pacientes con quemaduras en estado crítico. Dividieron a los sujetos en dos grupos, aquéllos con evidencia de lesión por inhalación en broncoscopia y aquéllos sin lesión. El punto final primario fue un compuesto de neumonía, lesión pulmonar aguda/síndrome de dificultad respiratoria aguda y muerte. ${ }^{14}$

\section{Scanning pulmonar con Xenón 133}

Estudio que nos permite valorar la relación ventilaciónperfusión mediante la inyección de Xenón 133, después de inyectar el hisopo se buscan áreas donde hay retención por más de 90 segundos. Tiene una certeza diagnóstica de $87 \%$ y se han observado alteraciones en la relación perfusión en obstrucción de la vía aérea parcial o total y de la circulación por Tromboembolia pulmonar. ${ }^{8}$

\section{Niveles de carboxihemoglobina}

La elevación en los niveles de carboxihemoglobina determina la lesión, niveles menores de $15 \%$ no presentan síntomas, 15-39\% producen cefalea, confusión, náusea, alteración del estado sensorio y letargo, y valores de $50-60 \%$ producen alteración del estado mental, alucinaciones, delirio y alteraciones cardiovasculares. ${ }^{8}$

\section{Gasometría arterial}

En la gasometría arterial podemos observar disminución de la presión parcial de oxígeno y elevación de la presión de dióxido de carbono, resulta un método sencillo y accesible. ${ }^{8}$

\section{Broncoscopia flexible, el estándar de oro en la lesión por inhalación}

La broncoscopia sigue siendo el estándar de oro en el diagnóstico de lesión por inhalación. Con esta técnica la lesión por inhalación se diagnostica por la presencia de eritema, acúmulos de hollín, ulceración o edema. ${ }^{15}$

Tiene una precisión de $86 \%$, mediante este método se facilita la intubación orotraqueal, aspiración y lavado bronquial de secreciones, extracción de tapones de moco y restos carbonáceos. ${ }^{8}$

Se ha propuesto una clasificación para valorar el grado de lesión mediante la broncoscopia flexible (Tabla 5).4,6

Por otra parte, la broncoscopia flexible nos puede ayudar a predecir la evolución y pronóstico de los pacientes en cuanto al daño respiratorio y detectar de forma temprana el desarrollo de neumonía (Figura 1). ${ }^{16}$

Tabla 5: Criterios por broncoscopia para la estratificación de la quemadura y lesión por inhalación.

\begin{tabular}{ccl}
\hline Grado & Lesión & Características \\
\hline 0 & No & $\begin{array}{l}\text { Ausencia de depósitos de carbón, } \\
\text { edema, broncorrea u obstrucción } \\
\text { Áreas leves, irregulares, de eritema, } \\
\text { depósitos de carbón en bronquios } \\
\text { proximales } \\
\text { Depósitos carbonatos y eritema } \\
\text { moderado, broncorrea, con o sin } \\
\text { compromiso de bronquios } \\
\text { Inflamación severa con depósitos } \\
\text { carbonosos abundantes, y tejido } \\
\text { friable, broncorrea y obstrucción } \\
\text { bronquial } \\
\text { Abundante desprendimiento } \\
\text { de mucosa, zonas de necrosis, } \\
\text { obliteración intraluminal }\end{array}$ \\
\hline
\end{tabular}



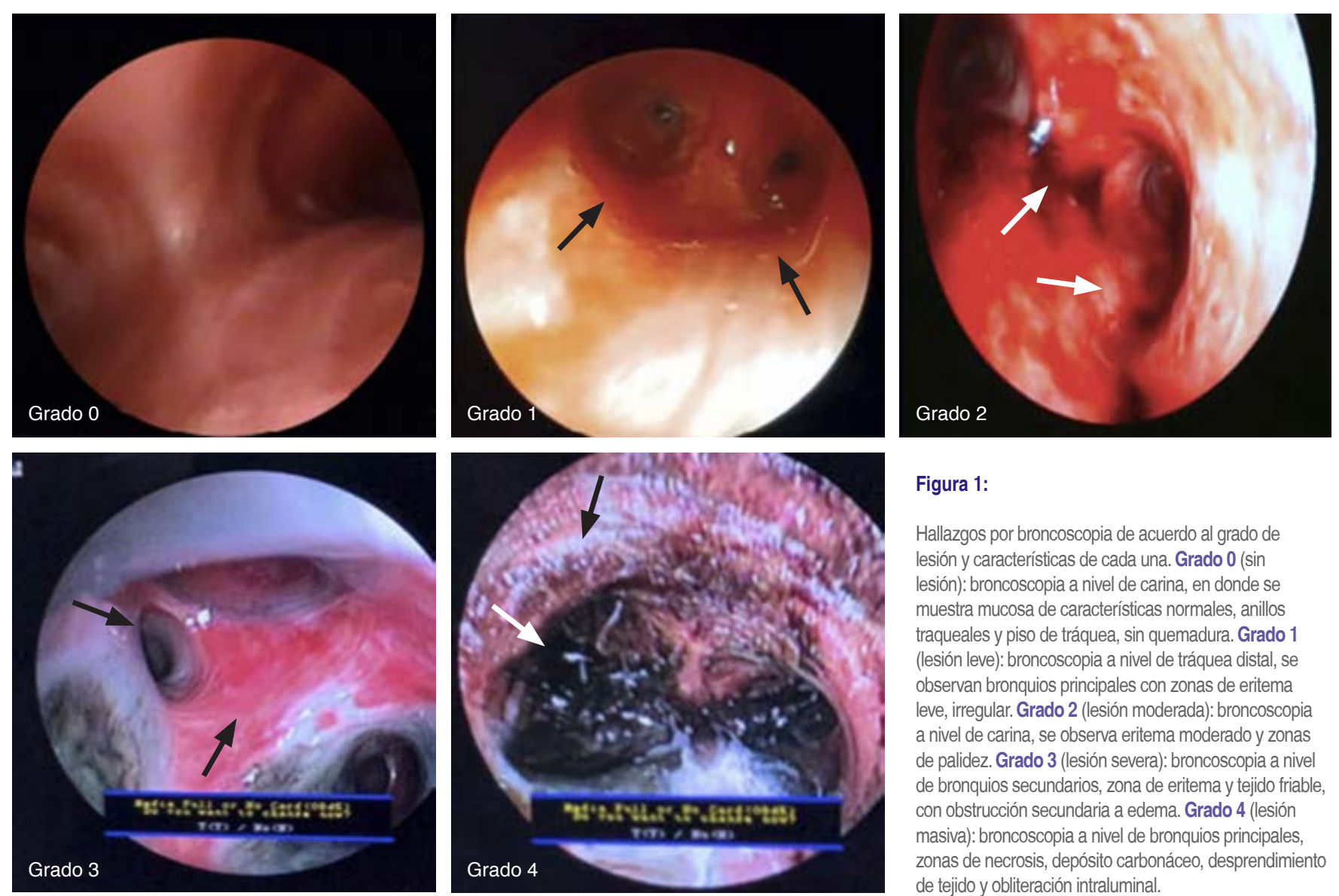

Figura 1:

Hallazgos por broncoscopia de acuerdo al grado de lesión y características de cada una. Grado 0 (sin lesión): broncoscopia a nivel de carina, en donde se muestra mucosa de características normales, anillos traqueales y piso de tráquea, sin quemadura. Grado 1 (lesión leve): broncoscopia a nivel de tráquea distal, se observan bronquios principales con zonas de eritema leve, irregular. Grado 2 (lesión moderada): broncoscopia a nivel de carina, se observa eritema moderado y zonas de palidez. Grado 3 (lesión severa): broncoscopia a nivel de bronquios secundarios, zona de eritema y tejido friable, con obstrucción secundaria a edema. Grado 4 (lesión masiva): broncoscopia a nivel de bronquios principales, zonas de necrosis, depósito carbonáceo, desprendimiento de tejido y obliteración intraluminal.

\section{TRATAMIENTO Y CONTROVERSIAS}

El paciente quemado con lesión por inhalación de la vía aérea debe recibir un manejo integral, representando retos y consideraciones especiales tanto en el tratamiento inicial como durante toda su estancia intrahospitalaria. Teniendo en cuenta que el manejo debe ser pronto y agresivo en la mayoría de los casos para evitar el riesgo de presentar un potencial desastre (Algoritmo 2).

\section{MANEJO DE LA VÍA AÉREA E INTUBACIÓN TEMPRANA}

La vía aérea en el paciente quemado será considerada de inicio una vía aérea difícil, ya que si la lesión involucra cara y cuello, la inflamación genera una distorsión de los tejidos, sangrado, exudado o secreciones, lo que dificulta el sello hermético para la ventilación con mascarilla facial, asimismo, se altera la identificación de las estructuras anatómicas durante la laringoscopia. Otra complicación que puede aparecer es la disminución en la movilidad de la mandíbula y cuello. ${ }^{17}$

La vía aérea de un paciente quemado en la fase de reanimación (primeras 48 horas) y fase aguda
(48 horas hasta el cierre de la última cicatriz) es cambiante, es decir, el paciente puede o no tener predictores iniciales de vía aérea difícil, pero durante la evolución de la reanimación y tratamiento quirúrgico, habrá cambios físicos concernidos con el proceso de curación, estos cambios generan que una vía aérea fácil evolucione a difícil en pocas horas después del tratamiento. Es por esta razón que en el manejo inicial de la vía aérea del quemado se deberá considerar una intubación en el momento oportuno para preservar su permeabilidad (Tabla 6), mientras dura la distorsión e intentar su extubación en cuanto la clínica lo permita y en casos más severos incluso realizar traqueostomía. ${ }^{17,18}$

Aunque ciertos estudios apoyen el uso de ventilación mecánica invasiva e intubación orotraqueal como profilaxis en la lesión pulmonar aguda, existen estudios en los que se apoya la aplicación de soporte con ventilación mecánica no invasiva, con los modos adecuados disminuye la necesidad de procedimientos invasivos en pacientes con lesión por inhalación. ${ }^{19}$

Existe mucha literatura acerca del manejo de la vía aérea en el paciente quemado; sin embargo, al final la decisión se debe tomar en torno a la valoración inicial de cada paciente. 


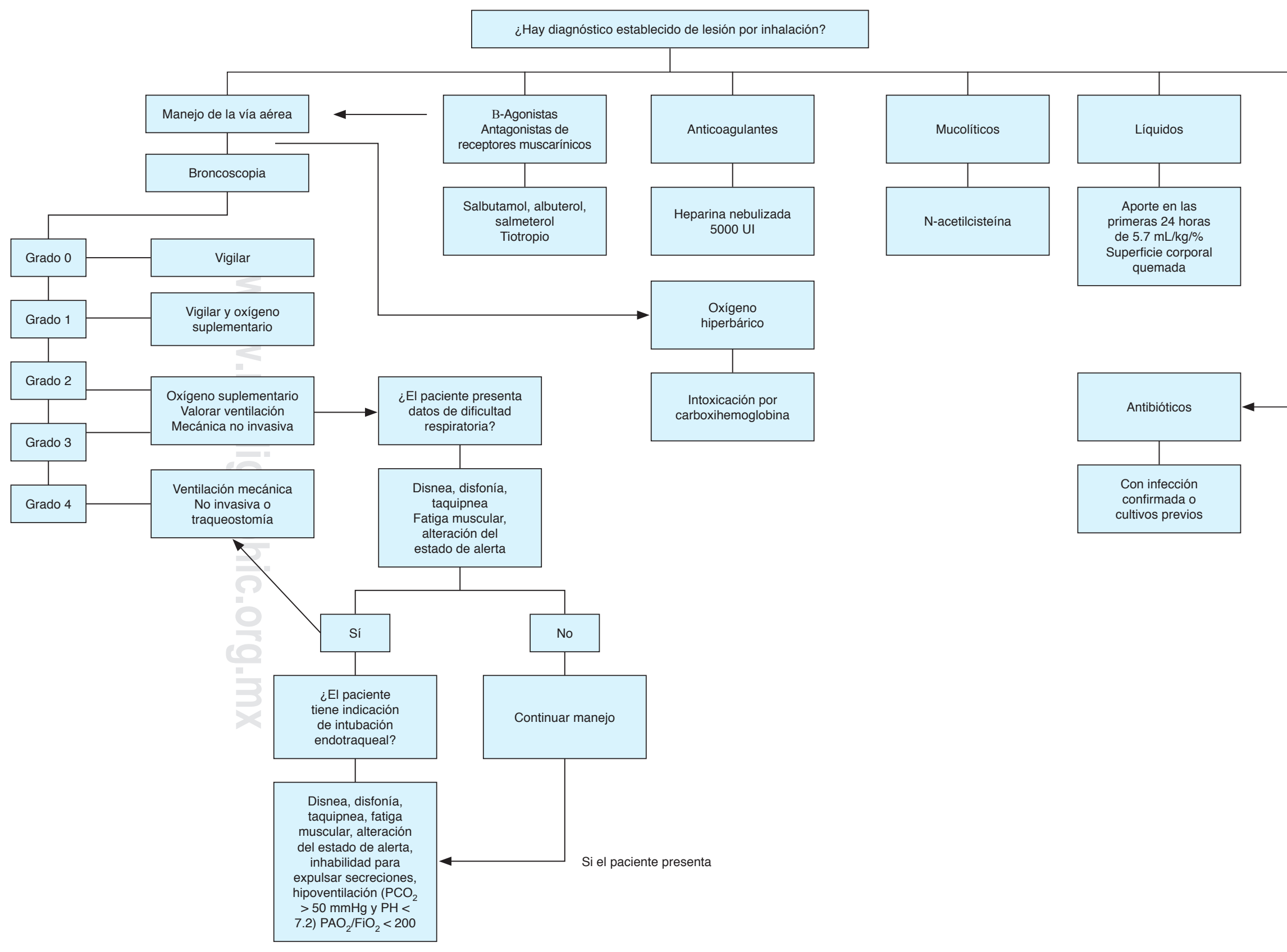

Algoritmo 2: Manejo del paciente con lesión por inhalación. 
Tabla 6: Indicaciones para intubación endotraqueal y ventilación mecánica.

- Obstrucción de la vía aérea superior

- Inhabilidad para expulsar secreciones

- Hipoxemia a pesar de oxígeno al $100 \%$

- Paciente con deterioro neurológico

- Fatiga muscular

- Hipoventilación $\left(\mathrm{PCO}_{2}>50 \mathrm{mmHg}+\right.$ y $\left.\mathrm{PH}<7.2\right)$

- ${ }^{*} \mathrm{PaO}_{2} / \mathrm{FIO}_{2}<200$

- Edema de la vía aérea superior

$\mathrm{mmHg}=$ milímetros de mercurio*

${ }^{*} \mathrm{PaO}_{2} / \mathrm{FiO}_{2}=$ Relación presión arterial de oxígeno/fracción inspirada de oxígeno.

\section{EFECTOS DE LA FLUIDOTERAPIA}

Resulta ser un punto importante de controversia, algunos autores prefieren un gran aporte de fluidos aun en presencia de edema pulmonar, ya que la pobre resucitación puede dar lugar a la aparición de fenómenos de isquemia local, que aumentan la respuesta inflamatoria, mientras que otros consideran que un aumento de este favorece el edema. ${ }^{10,20}$

Las observaciones clínicas sugieren que grandes volúmenes de resucitación, que a menudo se requieren para apoyar a los pacientes con quemaduras cutáneas, pueden acentuar el edema faríngeo que predispone la obstrucción aguda de la vía aérea superior. ${ }^{15}$

Se debe tomar en cuenta que si el paciente presenta quemadura corporal asociada, los requerimientos de líquidos aumentan, considerándose que los pacientes con inhalación requieren un aporte durante las primeras 24 horas de $5.7 \mathrm{~mL} / \mathrm{kg} / \%$ de superficie corporal quemada frente a $3.98 \mathrm{~mL} / \mathrm{kg} / \%$ superficie corporal quemada, en los pacientes sin lesión por inhalación. ${ }^{8}$

\section{B-AGONISTAS; UNA FORMA DE EVITAR BRONCOCONSTRICCIÓN}

La utilización de broncodilatadores es otro punto importante de controversia, ya que la resistencia de la vía aérea se produce principalmente por el edema de la mucosa, aunque se aconseja su utilización ya que los $\beta 2$ pueden activar el sistema mucociliar y aumentar el barrido de secreciones. ${ }^{8}$

Otros mecanismos que se plantean mediante la utilización de $\beta 2$ son que disminuyen la resistencia al flujo de aire, disminuyen la presión de la vía aérea por relajación del músculo liso limitando el grado de broncoespasmo y estimulan el aclaramiento mucociliar. ${ }^{6}$

\section{ANTAGONISTAS DE RECEPTORES MUSCARÍNICOS}

El mecanismo de acción de estos medicamentos está basado en que la respuesta parasimpática causa cons- tricción del músculo liso, al inhibir este efecto las presiones disminuyen y la secreción de moco y citocinas se reduce. ${ }^{6}$

En un modelo ovino se demostró que la combinación de quemadura e inhalación de humo, broncoespasmo y obstrucción de la vía aérea contribuyen a la insuficiencia pulmonar progresiva, fue un estudio aleatorizado, prospectivo de 36 ovejas, en el que se inició terapia con un antagonista de receptor muscarínico con bromuro de tiotropio (antagonista de receptor muscarínico M1 y M2) observando que disminuía la respuesta constrictiva de la vía aérea y obstrucción bronquial aguda para mejorar la función pulmonar. ${ }^{21}$

\section{AGENTES MUCOLÍTICOS: EL BENEFICIO BUSCADO}

El aclaramiento o limpieza en la vía aérea es un componente esencial en el manejo de la lesión por inhalación. Ésta se puede realizar mediante medidas mecánicas como tos terapéutica, fisioterapia de tórax, aspiración de secreciones, deambulación temprana y lavado de secreciones mediante broncoscopia. ${ }^{22}$

Dentro de las medidas farmacológicas, se ha estudiado el beneficio de la $\mathrm{N}$-acetilcisteína, ya que es considerado un potente agente mucolítico y tiene un papel en la mitigación de sustancias reactivas de oxígeno, además de ayudar a romper secreciones espesas de las vías respiratorias. Sin embargo, a su vez resulta ser un irritante de las vías respiratorias y puede producir broncoconstricción, por lo tanto es necesario que los pacientes sean predosificados con un broncodilatador. ${ }^{6}$

\section{ANTICOAGULANTES INHALADOS ¿UNA NOVEDAD?}

Los anticoagulantes inhalados se han utilizado para disminuir la formación de tapones de fibrina.

El primer estudio que se reportó acerca del beneficio de estos medicamentos fue un estudio de 90 pacientes pediátricos entre los años 1985-1995 que tenían una lesión por inhalación diagnosticada por medio de broncoscopia y que requirieron soporte ventilatorio. Este estudio constó de 43 controles y 47 pacientes que recibieron 5,000 UI de heparina nebulizada y $3 \mathrm{~mL}$ de $\mathrm{N}$-acetilcisteína al $20 \%$ cada cuatro horas los primeros siete días. Se observó una disminución significativa en la incidencia de reintubación por insuficiencia pulmonar progresiva y una reducción en la mortalidad. ${ }^{23}$

Posteriormente se realizó una revisión retrospectiva en adultos en la cual se llegó a la conclusión de que no se observó diferencia en el uso de heparina nebulizada en cuanto a los resultados en la lesión por inhalación; sin embargo, esto no es pauta para no utilizar esta medida. ${ }^{24}$ 
En cuanto a los efectos secundarios por el uso de anticoagulantes inhalados, se ha descrito un reporte de caso en un paciente pediátrico acerca de la coagulopatía asociada a heparina inhalada y terapia con $\mathrm{N}$-acetilcisteína, aunque los resultados no son concluyentes para contraindicar esta medida. ${ }^{25}$

En una revisión sistemática se confirmó que el uso de anticoagulantes inhalados, que incluyen heparina, heparinoides, antitrombina y fibrinolíticos, presentó una mayor supervivencia y disminución en la mortalidad. ${ }^{26}$

\section{ANTIBIÓTICOS, NO TODO ES LO QUE PARECE}

El uso profiláctico de antibióticos no está indicado, ya que no ofrece ningún beneficio en la prevención de la infección pulmonar y al contrario altera la competencia bacteriana facilitando el crecimiento y las infecciones por otros gérmenes. ${ }^{8}$

En una revisión sistemática en la que se evaluó los efectos de la profilaxis antibiótica, se analizaron 36 estudios controlados aleatorizados, en los que 26 evaluaron antibióticos tópicos, siete evaluaron antibióticos sistémicos (cuatro en el perioperatorio y tres desde la admisión), en dos se evaluaron antibióticos no absorbibles y un antibiótico local administrado por vía aérea. La profilaxis sistémica en pacientes quirúrgicos se evaluó en tres ensayos (119 pacientes), no hubo pruebas del efecto sobre las tasas de infección de la herida por quemaduras, los antibióticos sistémicos se relacionaron con una disminución significativa de la neumonía pero no de la sepsis.

En cuanto a la profilaxis perioperatoria y descontaminación selectiva con antibióticos no absorbibles, no tuvo efecto significativo.

Por otra parte, 11 estudios evaluaron la profilaxis tópica con sulfadiazina de plata y hubo aumento estadísticamente significativo en la infección de la herida por quemadura asociada a sulfadiazina de plata en comparación con los apósitos/sustitutos de piel, así como una estancia hospitalaria más larga. ${ }^{27}$

Las conclusiones que podemos extraer con respecto a los efectos de los antibióticos profilácticos en personas con quemaduras están limitadas por el volumen y la calidad de la investigación existente, por esto y siendo que éste es un tema de controversia relevante, el uso de antibióticos se ha reservado a la toma de cultivos previos.

\section{CORTICOSTEROIDES, EL TEMOR DE TODO MÉDICO}

Sabiendo precisamente que los corticosteroides resultan ser medicamentos muy efectivos en ciertas patologías, así como también muy temidos en el contexto de otras, en el paciente quemado no brindan ningún beneficio y al contrario aumentan la mortalidad.
Existen estudios donde se han reportado beneficios al disminuir la mortalidad pero únicamente realizados en animales. $8,10,28$

\section{OXÍGENO HIPERBÁRICO}

Indicado en lesiones donde se sospeche de concentraciones de carboxihemoglobina de $30 \%$. Puede reducir la vida media de las carboxihemoglobina a aproximadamente 20 minutos. ${ }^{6,29}$

\section{CONCLUSIONES Y RECOMENDACIONES}

- La lesión por inhalación es un término inespecífico que se refiere al daño del tracto respiratorio o parénquima pulmonar por calor, humo o irritantes químicos.

- La lesión por inhalación causa toxicidad sistémica debido a los gases tóxicos.

- La intubación está justificada en cualquier paciente con lesión por inhalación y signos de distrés respiratorio.

- Los broncodilatadores en aerosol son efectivos.

- Considerar la intubación profiláctica de acuerdo a la valoración integral de cada paciente.

- La limpieza de las vías respiratorias es un componente esencial en el tratamiento de los pacientes con lesión por inhalación.

- La ventilación mecánica de los pacientes con lesión por inhalación debe comenzar con volúmenes bajos.

- La ventilación mecánica no invasiva ha mostrado beneficios en el avance a ventilación mecánica invasiva.

- Considerar al paciente con lesión por inhalación como vía aérea difícil.

- La broncoscopia continúa siendo el estándar de oro diagnóstico y tratamiento en la lesión por inhalación.

- La lesión por inhalación se considera un factor pronóstico independiente en la evolución del paciente con lesión por inhalación.

- El papel de los urgenciólogos, intensivistas y anestesiólogos es primordial en la valoración inicial y diagnóstico temprano de la lesión por inhalación.

\section{REFERENCIAS}

- Zaragoza-Cárdenas PE. Manejo perioperatorio del paciente con quemadura de vía aérea y lesión por inhalación de humo. Rev Mex Anest. 2009;32(S1):S113-S116.

2. Vázquez-Torres J. Retos en el diagnóstico y manejo de la lesión por inhalación. Rev Mex Anest. 2014;37(Supl. 1):S218-S221.

3. Moctezuma-Paz LE, Páez-Franco I, Jiménez-González S, Miguel-Jaimes KD, Foncerrada-Ortega G, Sánchez-Flores AY, et al. Epidemiología de las quemaduras en México. Rev Esp Méd Quir. 2015;20:78-82.

4. Bai C, Huang H, Yao X, Zhu S, Li B, Hang J, et al. Application of flexible bronchoscopy in inhalation lung injury. Diagn Pathol. 2013;8:174.

5. Woodson L, Talon MD, Traber DL, Herndon DN. Diagnosis and treatment of inhalation injury. ${ }^{\odot} 2012$ Elsevier Ltd, Inc., BV. doi: 10.1016/B978-1-4377-2786-9.00019-9. 
6. Walker PF, Buehner MF, Wood LA, Boyer NL, Driscoll IR, Lundy $\mathrm{JB}$, et al. Diagnosis and management of inhalation injury: an updated review. Crit Care. 2015;19:351.

7. Rehberg S, Maybauer MO, Enkhbaatar P, Maybauer DM, Yamamoto Y, Traber DL. Pathophysiology, management and treatment of smoke inhalation injury. Expert Rev Respir Med. 2009;3(3):283-297.

8. Zapata SR, Cedeño D. Lesión inhalatoria. Fisiopatología, clínica, diagnóstico y tratamiento. Cir Plast Reconst Venez. 2004;6(12):53-62.

9. Grande CM, Myers RAM. Hiperbaric medicine: a trauma perspective. In: Stene JK, Grande CM, editors: Trauma anesthesia. Baltimore; Williams $\delta$ Wilkins. 1991, pp. 368-406.

10. Palomar M, Mosclans JR. Afectación de la vía aérea superior por quemadura e inhalación. En: Lorente JA., Esteban A. (eds). Cuidados Intensivos del paciente quemado. Barcelona, SpringerVerlay Ibérica, 1998, pp. 80-88.

11. Butte BJ, Butte BK. Quemaduras de vía aérea. Cuad Cir. 2002;16:69-76.

12. Peitzman AB, Shires GT, Teixidor HS, Curreri PW, Shires GT JR. Smoke inhalation injury: Evaluation of radiographic manifestations and pulmonary dysfunction. J Trauma. 1989;29:1232-1239.

13. Park MS, Cancio LC, Batchinsky AL, McCarthy MJ, Jordan BS, Brinkley WW, et al. Assessment of severity of ovine smoke inhalation injury by analysis of computed tomographic scans. $J$ Trauma. 2003;55:417-427.

14. Oh JS, Chung KK, Allen A, Batchinsky AL, Huzar T, King BT, et al. Admission chest CT complements fiberoptic bronchoscopy in prediction of adverse outcomes in thermally injured patients. $J$ Burn Care Res. 2012;33:532-538.

15. Haponik EF, Meyers DA, Munster AM, Smith PL, Britt EJ, Wise $\mathrm{RA}$, et al. Acute upper airway injury in burn patients. Serial changes of flow-volume curves and nasopharyngoscopy. Am Rev Respir Dis. 1987;135:360-366.

16. Amin M, Shaarawy H, El-Rab EG. Role of fiberoptic bronchoscopy in management of smoke inhalation lung injury. Egyptian Journal of Chest Diseases and Tuberculosis. 2015;64:733-737.

17. García-Lara MA. Vía aérea difícil del paciente gran quemado y con secuelas de quemaduras. Anestesia en el paciente quemado grandes retos en anestesia II. Rev Mex Anest. 2014;37(S1):S222-S225.

18. Rosen's emergency medicine: concepts and clinical practice, Ninth Edition. Quemaduras térmicas parte II. Rosen 2017, pp. 721-724.

19. Afife Ayla Kabalak, Ahmet Cınar Yasti. Management of inhalation injury and respiratory complications in Burns Intensive Care Unit. Ulus Travma Acil Cerrahi Derg. 2012;18(4):333-338.
20. Gordo F, Lorente FA, Esteba A. Alteraciones de la circulación pulmonar en el paciente gran quemado. En: Lorente SA, Esteban A (eds). Cuidados Intensivos del paciente quemado. Barcelona, Springer-Verlay Ibérica, 1998, pp. 341-352.

21. Jonkam C, Zhu Y, Jacob S, Rehberg S, Kraft E, Hamahata A, et al. Muscarinic receptor antagonist therapy improves acute pulmonary dysfunction after smoke inhalation injury in sheep. Crit Care Med. 2010;38(12):2339-2344 .

22. Carr JA, Crowley N. Prophylactic sequential bronchoscopy after inhalation injury: results from a three- year prospective randomized trial. Eur J Trauma Emerg Surg. 2013;39:177-183.

23. Desai MH, Mlcak R, Richardson J, Nichols R, Herndon DN. Reduction in mortality in pediatric patients with inhalation injury with aerosolized heparin/N-acetylcystine [correction of acetylcystine] therapy. J Burn Care Rehabil. 1998;19:210-222.

24. Holt J, Saffle JR, Morris SE, Cochran A. Use of inhaled heparin/ $\mathrm{N}$-acetylcystine in inhalation injury: does it help? J Burn Care Res. 2008;29(1):192-195.

25. Chopra A, Burkey B, Calaman S. A case report of clinically significant coagulopathy associated with aerosolized heparin and acetylcysteine therapy for inhalation injury. Burns. 2011;37:e73-e75.

26. Miller AC, Elamiri EM, Suffredini AR. Inhaled anticoagulation regimens for the treatment of smoke inhalation-associated acute lung injury: a systematic review. Crit Care Med. 2014;42(2):413419.

27. Barajas-Nava LA, López-Alcalde J, Roqué i Figuls M, Solà I, Bonfill Cosp X. Antibiotic prophylaxis for preventing burn wound infection. Cochrane Database Syst Rev. 2013;6(6):CD008738. doi: 10.1002/14651858.CD008738.pub2.

28. Haponik E, Summer WR. Respiratory complications in burn patients II. Diagnosis, management. J Crit Care. 1987;2:121-143.

29. Kim Y, Kym D, Hur J, Yoon J, Yim H, Cho YS, et al. Does inhalation injury predict mortality in burns patients or require redefinition? PLoS One. 2017;12(9):e0185195.

\author{
Correspondencia: \\ Marco Antonio Garnica Escamilla \\ Centro Nacional de Investigación \\ y Atención de Quemados (CENIAQ) INR LGII. \\ Calz. México Xochimilco Núm. 289, \\ Col. Arenal de Guadalupe, 14389, \\ Alcaldía Tlalpan. \\ E-mail: teranestmarco@yahoo.com.mx
}

